\title{
EMISSION LINES IN STAR FORMING GALAXIES
}

GUSTAVO BRUZUAL A.

Landessternwarte Heidelberg-Königstuhl

69117 Heidelberg, Germany

\author{
AND \\ GLADIS MAGRIS C. \\ Centro de Investigaciones de Astronomia (CIDA) \\ Apartado Postal 264, Mérida, Venezuela
}

\section{Abstract}

Magris et al. (1993, Proceedings of the ESO/OHP Workshop on Dwarf Galaxies, ed. G. Meylan) have combined the spectral evolution population synthesis code of Bruzual \& Charlot (1993, ApJ 405, 538) with the multipurpose photoionization-shock code MAPPINGS (Binette et al. 1993, AJ $105,797)$ in order to calculate self-consistently the evolution of the nebular emission corresponding to the ionizing spectra produced by the synthetic population. In this poster we compare the redshift dependence of the EW[OII]3727 predicted by the Magris et al. models with the observations available (Colles et al. 1990, MNRAS 244, 408; Kennicutt 1992, ApJS 79, 255; Le Fevre et al. 1994, ApJ 423, L89). Models in which stars form following the Salpeter IMF according to the SFR $\Psi(t)=1 M_{\odot} \tau^{-1} \exp (-t / \tau)$, for $\tau=1,2,3,5,7$, and $\infty$ Gyr have been considered. We assume $H_{0}=$ $50, \Omega=0, t_{g}=16 \mathrm{Gyr}$. About $\frac{2}{3}$ of the galaxies in these samples have EW[OII] 3727 from 2 to 5 times larger than the ones predicted by our models for steadily decreasing or even constant $(\tau=\infty)$ SFR. To cover the range of observed values of EW[OII]3727 we assume that instantaneous bursts (Salpeter IMF) of star formation take place in these galaxies, superimposed on the steady SFR used in the models. The mass added by the bursts as newly formed stars per unit mass of stars in the underlying galaxy is in the range $1-4 \times 10^{-4}$. Depending on the rest-frame wavelength, the color of the galaxies may remain unaffected or become considerably bluer as a consequence of the bursting activity. 